\title{
The impact of COVID-19 on palliative care workers across the world and measures to support their coping capacity
}

\section{Letter to the Editor}

Cite this article: Reynolds A, Hamidian Jahromi A (2021). The impact of COVID-19 on palliative care workers across the world and measures to support their coping capacity. Palliative and Supportive Care 19, 778-779. https://doi.org/10.1017/S1478951521000717

Received: 1 April 2021

Revised: 26 May 2021

Accepted: 30 May 2021

Author for correspondence:

Alireza Hamidian Jahromi, Plastic and Reconstructive Surgery Department, Rush University Medical Center, Chicago, IL, USA.

E-mail: alirezahamidian@yahoo.com (c) The Author(s), 2021. Published by Cambridge University Press
Allie Reynolds, B.A. ${ }^{1}$ (D) and Alireza Hamidian Jahromi, M.D., M.R.C.S. ${ }^{2}$ (D)

${ }^{1}$ Princeton University, Princeton, NJ and ${ }^{2}$ Plastic and Reconstructive Surgery Department, Rush University Medical Center, Chicago, IL

We read with great interest the recently published article by Pastrana et al. (2021) highlighting ways in which palliative care workers (PCWs) have been impacted by the COVID-19 pandemic and coping strategies they've adopted. In this study, the authors urged hospitals and healthcare authorities to implement measures to support PCW's coping capacity. While we commend the authors for their valuable findings and discussions, we would like to propose additional recommendations for coping strategies geared toward PCW.

One way in which PCW can positively impact their mental health is by boosting emotional connectedness. A recent study described the relationship between emotional connectedness and mental health as well as a variety of strategies healthcare workers (HCWs) can use to stay emotionally connected with others during the pandemic (Bender et al., 2021). Since the pandemic has persisted and will continue to do so in the foreseeable future, boosting HCW's emotional connectedness needs to become a priority.

The pandemic is a stressful time for all, and even more so for PCW. There are numerous ways in which healthcare settings can assist with stress management in PCW. The first step in boosting emotional connectedness and reducing stress is to entitle all PCW to more frequent and longer break times throughout their shifts. Designated break times can offer PCW valuable opportunities to relax and destress, especially when they are exposed to numerous stressful events during the day. Management teams can also set programs to create bonds among workers, which Bender et al. showed to be extremely important for emotional connectedness, that occur virtually or in person using personal protective equipment. Examples of such programs could be workout or cooking competitions (adding an extra element of competition as a motivating factor) and sharing pictures or stories about past travels among a shared community.

Physical health is also a strong contributor to mental health (Ohrnberger et al., 2017), so management teams should add an extra focus on PCW to stay active, eat healthy, and get enough sleep despite their long and stressful shifts. All of these suggestions can be implemented on the healthcare setting level and to the PCW community as a whole.

There are currently several COVID-19 vaccines available to healthcare professionals and will soon be offered to the general public. The American public's opinion regarding vaccination against COVID-19 is hesitant in general, but it is important that the majority of the population is vaccinated to prevent further spread (Dror et al., 2020; Reiter et al., 2020). PCW, as well as other HCW, are in a unique position of authority to advocate for vaccination and in the past have acted as strong influencers in the general public's vaccine decisions (Paterson et al., 2016). Bringing people together is a meaningful way of boosting emotional connectedness and promoting COVID-19 vaccination could be a valuable way to do so for PCW and HCW overall. Presenting the HCW as a united front in favor of COVID-19 vaccination allows for expanded interpersonal relationships and could also result in more favorable opinions among the general public toward COVID-19 vaccination.

As the COVID-19 pandemic continues, the work-related stress of PCW will likely continue to increase. We suggest that hospitals and other healthcare settings provide tangible ways for PCW to boost their emotional connectedness and mental health during this especially stressful time. Proposing additional adaptive strategies for coping with pandemic stress will be of the utmost importance for improving PCW's mental health during this time.

\section{Conflict of interest}

None.

\section{References}

Bender AE, Berg KA, Miller EK, et al. (2021) "Making sure we are all okay": Healthcare workers' strategies for emotional connectedness during the COVID-19 pandemic. Clinical Social Work Journal. doi:10.1007/ s10615-020-00781-w 
Dror AA, Eisenbach N, Taiber S, et al. (2020) Vaccine hesitancy: The next challenge in the fight against COVID-19. European Journal of Epidemiology 35(8), 775-779. doi:10.1007/s10654-020-00671-y

Ohrnberger J, Fichera E and Sutton M (2017) The relationship between physical and mental health: A mediation analysis. Social Science \& Medicine 195, 42-49. doi:10.1016/j.socscimed.2017.11.008

Pastrana T, De Lima L, Pettus K, et al. (2021) The impact of COVID-19 on palliative care workers across the world: A qualitative analysis of responses to open-ended questions. Palliative \& Supportive Care. doi:10.1017/ S1478951521000298

Paterson P, Meurice F, Stanberry LR, et al. (2016) Vaccine hesitancy and healthcare providers. Vaccine 34(52), 6700-6706. doi:10.1016/j.vaccine. 2016.10.042

Reiter PL, Pennell ML and Katz ML (2020) Acceptability of a COVID-19 vaccine among adults in the United States: How many people would get vaccinated? Vaccine 38(42), 6500-6507. doi:10.1016/j.vaccine.2020.08.043 